\title{
Clinical Outcomes and Economic Impact of Oritavancin for Gram-Positive Infections: A Single Academic Medical Center Health System Experience
}

\author{
Lauren E. Brownell ${ }^{1}$ Meagan L. Adamsick ${ }^{2}$ - Erin K. McCreary ${ }^{3}$. Joshua P. Vanderloo ${ }^{1}$ - Erika J. Ernst ${ }^{4}$. \\ Emily R. Jackson ${ }^{1} \cdot$ Lucas T Schulz $^{1}$
}

Published online: 26 June 2020

(c) The Author(s) 2020

\begin{abstract}
Background Vancomycin treatment of complicated Gram-positive infections is associated with laboratory monitoring, nephrotoxicity, and multiple daily dosing. Oritavancin, a lipoglycopeptide antibiotic with a once-weekly dosing strategy and similar but slightly broader spectrum of activity, presents several opportunities over vancomycin to improve compliance and convenience for the patient. Minimal real-world clinical and acquisition cost data in the inpatient setting and clinical data surrounding multiple dosing in the outpatient setting have limited oritavancin use despite its potential logistic advantages. Objectives We describe inpatient and outpatient oritavancin administration, clinical outcomes, and economic impact. Methods This was a single-center, retrospective case series of patients treated with at least one dose of oritavancin between May 2015 and September 2017 at an academic medical center in the USA. A simplified cost-avoidance analysis was conducted assuming the patient had a national health insurance plan and focused on hospital days prevented.

Results Seventy-five patients received oritavancin during the study period. The most common use of oritavancin was in patients with acute bacterial skin and skin structure infections (ABSSSI), defined as cellulitis, abscess or non-surgical wounds $(n=25,33 \%)$, followed by surgical wound infections $(n=12,16 \%)$ and osteomyelitis or septic arthritis $(n=10,13 \%)$. Clinical cure or improvement was achieved in 68 patients $(93.2 \%)$, while five patients (6.8\%) failed treatment; adverse reactions were reported in nine patients (12\%). Thirty-five patients received oritavancin as inpatients; 20 patients (57\%) had at least one hospital day avoided due to inpatient oritavancin administration resulting in a total cost avoidance of US\$343,654.

Conclusion In this series of 75 patients with Gram-positive infections, oritavancin treatment resulted in clinical cure or improvement in most patients, and was generally well tolerated. Inpatient administration may avoid costs and outpatient administration is a reasonable consideration for patients in which prolonged antibiotic therapy is necessary.
\end{abstract}

\section{Introduction}

Digital features To view digital features for this article go to https ://doi.org/10.6084/m9.figshare.12221690

Lucas T Schulz

1schulz2@uwhealth.org

1 Department of Pharmacy, University of Wisconsin Health, 600 Highland Avenue, MC 1530, Madison, WI 53792, USA

2 Department of Pharmacy, Massachusetts General Hospital, Boston, MA, USA

3 Department of Pharmacy, University of Pittsburgh Medical Center, Pittsburgh, PA, USA

4 University of Iowa College of Pharmacy, Iowa City, IA, USA
The treatment of complex Gram-positive infections often requires prolonged courses of intravenous (IV) antibiotic therapy [1]. Vancomycin is the most common IV therapy prescribed, most often for infections due to methicillinresistant Staphylococcus aureus (MRSA). Vancomycin therapy is challenging due to dosing frequency, therapeutic drug monitoring, and drug-associated toxicities [2, 3]. Antibiotic agents that overcome these challenges are needed, particularly for infections that require a prolonged treatment course.

Oritavancin is a lipoglycopeptide antibiotic approved by the US Food and Drug Administration (FDA) in 2015 as a single-dose treatment for acute bacterial skin and skin structure infections (ABSSSI) [4-6]. Initial experiences 


\section{Key Points}

The majority (93.2\%) of patients with Gram-positive infections who were treated with oritavancin achieved clinical cure or improvement, and more than half (57\%) avoided at least one hospital day due to inpatient oritavancin treatment, resulting in US\$343,654 of avoided costs.

In certain populations where long-term IV line placement is not ideal, oritavancin could be used to facilitate care transition and hospital discharge. The rationale for oritavancin use in most patients was avoidance of longterm IV-line placement (61\%).

This analysis of real-world clinical and economic data indicate use of multiple-dose oritavancin is a safe and effective treatment option, and for patients who require prolonged antibiotic therapy, offers potential logistical, clinical, and cost benefits.

with oritavancin for non-FDA approved indications have been described in three case series [7-9]. Oritavancin use in complex, non-ABSSSI Gram-positive infections remains uncharacterized. Oritavancin use has been limited by concerns regarding high drug acquisition cost, limited inpatient reimbursement, and treatment-emergent osteomyelitis reported from phase 3 trials $[5,10]$.

We sought to describe real-world use at our institution in order to identify clinical outcomes and economic impact, particularly when oritavancin is used in the inpatient setting to facilitate transitions of care and hospital discharge.

\section{Methods}

This was a retrospective analysis of patients who received at least one dose of oritavancin at an academic medical center health system in the midwestern USA comprised of four hospitals and over 80 clinics. The electronic medical record (EMR) was queried for patients with an oritavancin order from May 2015 to September 2017. Seventeen previously reported patients were included in the analysis so that the economic impact of oritavancin utilization could be fully evaluated [9]. Patients were only excluded if oritavancin was ordered but not administered.

Two pharmacists conducted an independent and comprehensive chart review of each oritavancin administration. Patient demographics, oritavancin indication, and setting of oritavancin administration within the health system (i.e., inpatient, outpatient, or both) were collected. Indication was determined by the infectious disease (ID) consultation progress notes. If the patient did not have an
ID consultation note, the indication was determined by documentation provided by an ID-trained pharmacist in the oritavancin electronic prescription record.

Reviewers investigated oritavancin rationale for use, clinical outcomes, and safety. Oritavancin rationale for use was determined by EMR review of ID consultation and inpatient case management documentation and categorized as: IV-line placement avoidance due to history or active substance-use disorder, social or insurance barrier, allergy or adverse drug reaction to alternative Gram-positive agents, drug-drug interactions, medication non-adherence, or other.

Clinical cure was defined as resolution of signs and symptoms of infection without the need for further treatment after the completion of the oritavancin course [9]. Clinical improvement was defined as recovery from infectious signs and symptoms with the need for subsequent Gram-positive therapy after the completion of the oritavancin course. Clinical failure was defined by the progression of infectious signs and symptoms and the need for alternative Gram-positive therapy during the oritavancin course. Outcomes were determined by review of all clinical documentation following oritavancin therapy within the EMR, including subsequent clinic encounters and telephone records; most patient data were assessed within 28 days of the end of therapy.

Safety outcomes, reviewed independently of clinical outcomes, included adverse drug reactions (ADRs) to oritavancin and hospitalization within 30 days of receiving oritavancin. The Naranjo Adverse Drug Reaction Probability Scale (NADRPS) was used to determine the probability of the adverse event attributable to oritavancin [11].

The number of hospital days avoided was calculated for patients receiving oritavancin to facilitate discharge when no alternative therapy option was available (i.e., the patient required hospitalization to receive antimicrobial therapy or could receive oritavancin and discharge). Hospital days avoided was equal to the total course of IV antibiotics necessary minus the hospital day that oritavancin was administered. The total course of IV antibiotics was determined from specific recommendations indicated by the ID physician in the patient's chart, or when not specified in the chart, from national consensus guidelines.

A simplified cost-avoidance analysis was conducted for all patients with hospital days avoided by assuming a national health insurance plan. Inputs into the analysis included medication cost, hospitalization cost, hospital days avoided, and Medicare reimbursement for each indication. Hospitalization cost was set at \$2533 (US\$) per day [12]. Medicare diagnosis-related group (DRG) was assigned based on indication. Reimbursement data were extracted from the fiscal year 2015 Medicare Provider Charge Inpatient Data, sorted by DRG, filtered by Wisconsin, converted into 2018 dollar amounts using the United States Bureau of Labor inflation calculator, and then averaged to give an estimated reimbursement for 
each patient $[13,14]$. Wisconsin data were not available for endocarditis, so all national data were used for this indication and averaged. Average wholesale price (AWP) reported in 2018 was utilized for oritavancin cost. A positive return on investment (ROI) occurred when the money saved through hospital days avoided and Medicare reimbursement exceeded the cost of the hospitalization and inpatient oritavancin dose. If patients were re-admitted before the end of their projected treatment course, the days left in their treatment course was subtracted from their days saved, and the cost of their re-hospitalization was incorporated into the overall cost analysis.

\section{Results}

Eighty-one unique patients had oritavancin ordered between May 2015 and September 2017. Six patients never received the medication and were excluded. The study population included 75 patients receiving 187 doses administered (dose range per patient 1-32). All patients received an initial $1200 \mathrm{mg}$ dose followed by $1200 \mathrm{mg}$ or $800 \mathrm{mg}$ weekly; patients receiving multiple doses followed a previously published protocol [9]. Two patients were excluded from the efficacy analysis due to infusion reactions occurring during the first oritavancin dose, leading to termination of therapy; these patients were included in the safety analysis (Fig. 1).

Baseline demographic data are summarized in Table 1. Patients were predominately middle-aged (50 years, range 20-86 years), male (57.3\%), and included people who inject drugs (PWID) (13.3\%). The most common indications for oritavancin use were ABSSSI $(n=25,33 \%)$, surgical wound infections $(n=12,16 \%)$, and osteomyelitis/ septic arthritis $(n=10,13 \%)$. Seventeen patients received oritavancin without a culture, five $(6.6 \%)$ for a culture negative condition, and $53(70.7 \%)$ patients received culture-targeted therapy with a total of 73 pathogens. The most common pathogens were methicillin-susceptible Staphylococcus aureus (MSSA) $(n=23)$, MRSA $(n=13)$, coagulase-negative Staphylococcus $(n=4)$, and vancomycin-resistant Enterococcus faecium $(n=4)$. The frequency of pathogens is listed in Table 2.

Patients were most likely to receive oritavancin in the ambulatory setting alone $(n=40,35 \%)$. Twenty (27\%) patients received oritavancin in both inpatient and ambulatory settings, while 15 (20\%) patients received oritavancin only as an inpatient. Of patients receiving oritavancin only as an outpatient, 24 (60\%) came from the inpatient setting with an infectious diseases consult recommending outpatient oritavancin.

\subsection{Efficacy Analysis}

Of the 73 patients included in the efficacy analysis, 34 patients $(46.6 \%)$ experienced cure, 34 patients $(46.6 \%)$

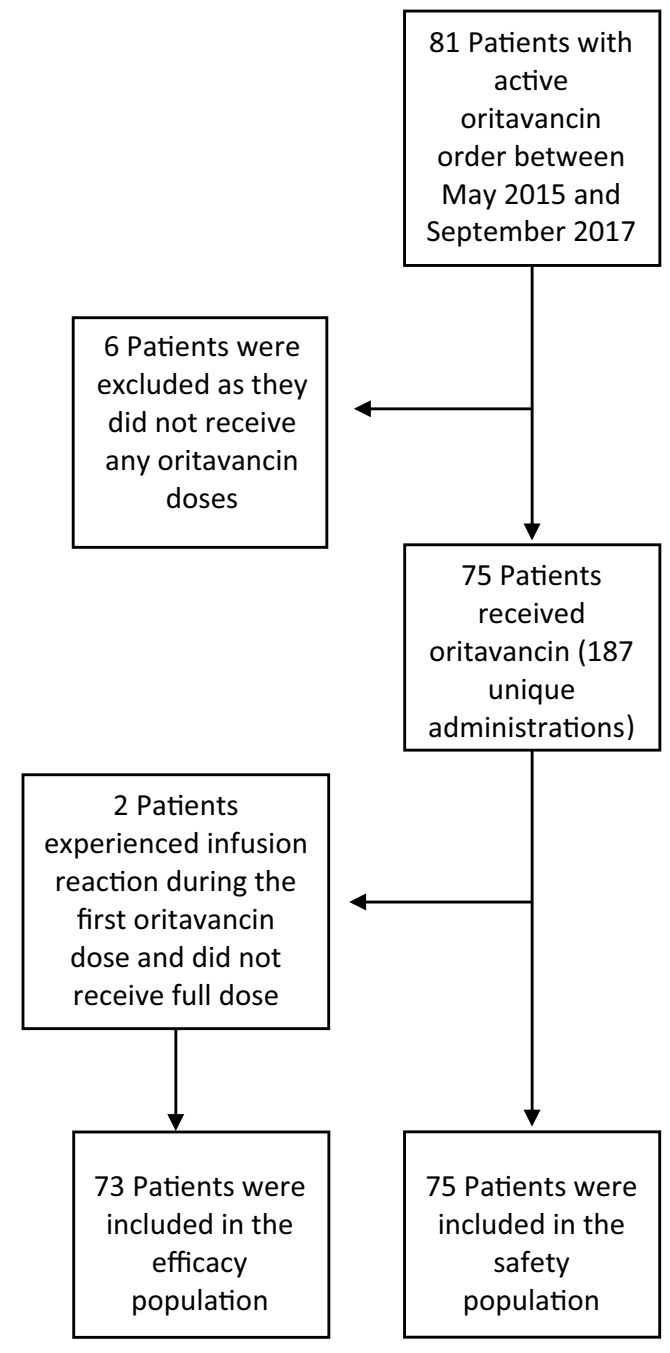

Fig. 1 Patients included in the safety and efficacy analysis

improved, and five patients $(6.8 \%)$ failed treatment (Table 1). Failure occurred in septicemia $(n=2)$, surgical infection $(n=1)$, ABSSSI $(n=1)$, and hepatic abscess $(n=1)$. The rationale for oritavancin use in most patients was avoidance of long-term IV-line placement (61\%) (Table 3).

We identified ten PWID who received multiple doses of oritavancin to avoid long-term IV-line placement; indications included osteomyelitis, prosthetic device infection, endocarditis, and surgical wound infection. Seven (70\%) of the PWID patient subgroup achieved cure or improvement.

\subsection{Safety Analysis}

Nine (12\%) ADRs were reported. Utilizing the NADRPS harm scale, these were further classified as one definite, seven probable, and one possible. Four (44.4\%) adverse events occurred during or after the second dose (back pain 
Table 1 Baseline demographic and efficacy and safety outcomes

\begin{tabular}{|c|c|c|c|c|c|c|}
\hline \multirow[t]{2}{*}{ Indication } & \multicolumn{3}{|c|}{ All patients $(n=75)$} & \multicolumn{3}{|c|}{ Patients included in efficacy analysis $(n=73)$} \\
\hline & $\begin{array}{l}\text { Age } \\
\text { (avg, } \\
\text { years) }\end{array}$ & Male, $n(\%)$ & $\begin{array}{l}\text { Weight (avg IBW, } \\
\mathrm{kg} \text {, avg ABW, kg) }\end{array}$ & $\begin{array}{l}\text { Clinical cure or } \\
\text { improvement }^{\mathrm{b}}, n \\
(\%)\end{array}$ & Failure, $n(\%)$ & $\mathrm{ADR}, n(\%)$ \\
\hline All indications & $\mathbf{5 0}$ & $43(57.3)$ & $85.1,65.6$ & $68(93.2)$ & $5(6.8)$ & $9(12)$ \\
\hline ABSSSI (all $n=25$; efficacy analysis $n=24$ ) & 54 & $15(60)$ & $66.8,92.8$ & $23(95.8)$ & $1(4.2)$ & $4(16.7)$ \\
\hline Diabetic foot infection $(n=3)$ & 56 & $2(66.7)$ & $69.2,100.4$ & $3(100)$ & $0(0)$ & $0(0)$ \\
\hline Endocarditis $(n=4)$ & 37 & $3(75)$ & $67.8,71.2$ & $4(100)$ & $0(0)$ & $0(0)$ \\
\hline Line infection $(n=2)$ & 38 & $1(50)$ & $66.2,55.5$ & $2(100)$ & $0(0)$ & $1(50)$ \\
\hline Other $^{\mathrm{a}}(n=5)$ & 53 & $1(20)$ & $62.7,93.3$ & $4(80)$ & $1(20)$ & $0(0)$ \\
\hline Osteomyelitis/septic arthritis $(n=10)$ & 54 & $5(50)$ & $61.8,83.4$ & $10(100)$ & $0(0)$ & $2(20)$ \\
\hline Pneumonia $(n=5)$ & 48 & $3(60)$ & $66.7,75.6$ & $5(100)$ & $0(0)$ & $0(0)$ \\
\hline $\begin{array}{l}\text { Prosthetic device infection (all } n=4 \text {; efficacy } \\
\text { analysis } n=3 \text { ) }\end{array}$ & 48 & $1(33.3)$ & $59.2,85.6$ & $3(100)$ & $0(0)$ & $1(33.3)$ \\
\hline Sepsis $(n=5)$ & 47 & $4(80)$ & $67.9,92.5$ & $3(100)$ & $2(40)$ & $0(0)$ \\
\hline Surgical wound infection $(n=12)$ & 45 & $8(66.7)$ & $66.6,76.5$ & $11(91.7)$ & $1(8.3)$ & $1(8.3)$ \\
\hline
\end{tabular}

$I B W$ ideal body weight, $A B W$ actual body weight, $A B S S S I$ acute bacterial skin and skin structure infections, $I V$ intravenous

${ }^{a}$ Other $=$ left parotid sialadenitis with right parotid duct abscess with a large sialolith, infection of trigeminal neurotrophic ulceration infected with methicillin resistant Staphylococcus aureus, hepatic abscess, tenosynovitis

b34 patients were defined as having clinical cure and 34 patients were defined as having clinical improvement; 34 patients with clinical improvement received oral antibiotics after administration of oritavancin as IV to oral step-down therapy

Table 2 Frequency of infecting pathogens targeted with oritavancin therapy

\begin{tabular}{lll}
\hline Pathogens & No. of isolates & $\%$ of isolates \\
\hline Total & $\mathbf{7 3}$ & $\mathbf{1 0 0 \%}$ \\
Methicillin-susceptible Staphylococcus aureus (MSSA) & 23 & $31.5 \%$ \\
Methicillin-resistant Staphylococcus aureus (MRSA) & 13 & $17.8 \%$ \\
Coagulase-negative Staphylococcus (CoNS) & 4 & $5.8 \%$ \\
Vancomycin-resistant Enterococcus faecium (VRE) & 4 & $5.8 \%$ \\
Enterococcus faecalis & 3 & $4.1 \%$ \\
Streptococcus agalactiae & 3 & $4.1 \%$ \\
Endogenous flora of the lungs & 3 & $4.1 \%$ \\
Streptococcus anginosis & 2 & $2.7 \%$ \\
Streptococcus viridans & 2 & $2.7 \%$ \\
Corynebacterium striatum & 2 & $2.7 \%$ \\
Mixed skin flora & 2 & $2.7 \%$ \\
Enterococcus faecium (VSE) & 1 & $1.4 \%$ \\
Enterococcus gallarium & 1 & $1.4 \%$ \\
Small colony variant MRSA & 1 & $1.4 \%$ \\
Streptococcus group G & 1 & $1.4 \%$ \\
Streptococcus mitis group & 1 & $1.4 \%$ \\
Corynebacterium species (not striatum) & 1 & $1.4 \%$ \\
Propionibacterium acnes & 1 & $1.4 \%$ \\
Propionibacterium species (not acnes) & 1 & $1.4 \%$ \\
Peptostreptococcus spp (unidentified) & 1 & $1.4 \%$ \\
Finegoldia magna & 1 & $1.4 \%$ \\
Lactobacillus spp & 1 & $1.4 \%$ \\
\hline & &
\end{tabular}


Table 3 Rationale for oritavancin use

\begin{tabular}{ll}
\hline Rationale for use & No. of patients (\%) \\
\hline IV-line placement avoidance & $46(61.3)$ \\
Social and/or insurance barriers & $36(46.7)$ \\
Allergy/ADR to alternative antibiotics & $15(20)$ \\
Other $^{\mathrm{a}}$ & $8(10.7)$ \\
Drug-drug interaction with alternative antibiotics $^{2}$ & $3(4)$ \\
\hline
\end{tabular}

Patients could have more than one rationale for use if it was documented within the electronic medical record

$A D R$ adverse drug reaction, MRSA methicillin-resistant Staphylococcus aureus, VRE vancomycin-resistant Enterococcus

${ }^{\mathrm{a}}$ Other $=$ specific coverage for small colony variant MRSA $(n=1)$, broad Gram-positive coverage $(n=3)$, desire for long-acting coverage $(n=1)$, VRE resistant to meropenem and daptomycin $(n=1)$, rationale not documented within health record $(n=2)$

in two patients, neck pain, and fatigue). Overall, the most common reaction was back pain with infusion $(n=3)$. Others included rash, flushing, pruritus, headache, shortness of breath, and pancytopenia. Management of adverse drug reactions was consistent with previous literature; all patients experienced quick resolution (less than $120 \mathrm{~min}$ ) upon drug discontinuation [9]. Pancytopenia occurred in a female patient receiving oritavancin and ertapenem concomitantly. Both medications were held for 7 days while her cell counts recovered. Ertapenem monotherapy was restarted and cell counts remained stable; thus, the combination or oritavancin therapy received an NADRPS score of 5 (probable).

Eight patients (10.6\%) were hospitalized within 30 days of receiving oritavancin. Admission reasons were progressing infection ( $n=4)$, ADR to oritavancin $(n=3)$, and tenuous clinical status with no antibiotic changes $(n=1)$. Progressing infection leading to hospitalization was associated with ABSSSI $(n=1)$, endocarditis $(n=1)$, osteomyelitis/septic arthritis $(n=1)$, and hepatic abscess $(n=1)$. Shortness of breath was the adverse reaction resulting in admission for new pneumonia diagnosis evaluation.

\subsection{Cost Avoidance Analysis}

Forty patients received oritavancin exclusively in the outpatient setting and 15 patients received only inpatient administrations. Hospital days were avoided in 20 patients (57\%) (Table 4) who received inpatient administration prior to discharge and completion of therapy with outpatient treatment. Two patients were PWID and were re-admitted prior to the end of their treatment course. When a simplified cost-avoidance analysis was conducted, a positive return on investment (ROI) was found in three of seven surgical wound infections, one of six ABSSSIs, three of three endocarditis infections, one of three pneumonia infections, and one of one prosthetic device infections. There was no apparent relationship between a positive ROI and the hospital day of oritavancin administration. Inpatient oritavancin administration for patients with national health insurance and at least one hospital day avoided resulted in an estimated cost avoidance to the institution of $\$ 343,654$ for 73 patients over 2 years.

\section{Discussion}

We present the clinical outcomes and economic impact of 75 patients who received oritavancin for the treatment of Gram-positive infections. Our results show oritavancin is a safe and effective treatment option associated with potential cost-avoidance in certain patient populations.

Oritavancin is FDA approved for ABSSSI treatment, although off-label use has been previously described [7-9]. The primary indication for oritavancin in our patient

Table 4 Cost avoidance analysis of oritavancin in patients with hospital days saved

\begin{tabular}{lllll}
\hline Indication & $\begin{array}{l}\text { Average hospital day of } \\
\text { oritavancin administration; } \\
n \text { (range) }\end{array}$ & $\begin{array}{l}\text { Average hospital } \\
\text { days avoided; } n \\
\text { (range) }\end{array}$ & $\begin{array}{l}\text { Average cost avoidance } \\
\text { associated with inpatient } \\
\text { oritavancin administration; } \\
\text { US\$ amount }\end{array}$ & $\begin{array}{l}\text { Patients with a positive return } \\
\text { on investment per indication; } \\
n(\%)\end{array}$ \\
\hline All indications $(n=20)$ & $6(2-20)$ & $12(3-48)$ & 15,621 & $9(45)$ \\
Cellulitis $(n=6)$ & $5(2-9)$ & $6(3-7)$ & 5,252 & $1(16.7)$ \\
Endocarditis $(n=3)^{\mathrm{a}}$ & $7(5-11)$ & $18(14-26)$ & 47,246 & $3(100)$ \\
$\begin{array}{l}\text { Pneumonia }(n=3) \\
\begin{array}{c}\text { Prosthetic device infection } \\
(n=1)\end{array}\end{array}$ & $5(6-9)$ & $16(8-30)$ & 24,955 & $1(33.3)$ \\
$\begin{array}{c}\text { Surgical wound infection } \\
(n=7)^{\mathrm{a}}\end{array}$ & $7(3-20)$ & 10 & 19,221 & $3(100)$ \\
\hline
\end{tabular}

${ }^{\text {a }}$ Two patients (one with endocarditis and one with a surgical wound infection) with a history of being people who inject drugs (PWID) were readmitted prior to the end of their anticipated treatment course. One patient presented with back chest pain during the second oritavancin infusion. This patient was found to have a toxicology screen positive for cocaine and oritavancin was ultimately continued. The other patient presented 9 days following oritavancin infusion with bacteremia, thought to be due to continuing as a PWID. Antibiotic therapy was adjusted 
population was ABSSSI; these patients demonstrated clinical outcomes consistent with previous literature confirming usefulness of oritavancin for treatment of ABSSSI in a real-world setting $[4,5]$. Oritavancin was used for nonABSSSI infection types in 49 patients (67\%); our data suggest oritavancin is a safe and effective treatment option for complex Gram-positive infections, as $93.2 \%$ of patients had an outcome consistent with previous case series [7-9, 15].

Reported oritavancin adverse reactions include infusion reactions, anemia, and chest pain [4, 5, 9]. The SOLO clinical trial report identified six patients with osteomyelitis, coded as an adverse event, leading to a warning in the prescribing information about use of oritavancin in osteomyelitis [5]. All osteomyelitis cases were discovered within 9 days of oritavancin administration, suggesting the osteomyelitis may have been subacute at the time of oritavancin administration. In our report, ten patients with osteomyelitis or septic arthritis were treated with oritavancin and all patients achieved favorable clinical outcomes. Further exploration of the efficacy of oritavancin for osteomyelitis is warranted, however; these data suggest oritavancin as a practical therapy option [16].

Central venous catheter avoidance in the PWID population is controversial and PWID patients often must remain hospitalized or under observed care for the duration of IV antibiotic treatment [17]. Oritavancin requires a peripheral IV catheter to be placed for administration, likely once weekly, instead of a central catheter. A published single case series of oritavancin use in the PWID patient population included four PWIDs with one clinical cure, two clinical failures, and one patient with an unevaluable outcome [15]. The SOLO clinical trials included a $30 \%$ PWID population $[4,5]$. In our study a high clinical cure or improvement was achieved; real-world data should continue to be collected to determine optimal use of oritavancin in the PWID population.

Notably, treatment paradigms for two included disease states (endocarditis and osteomyelitis) may be shifting away from long-term parenteral therapy. Recently published trials in osteomyelitis and endocarditis demonstrate successful clinical outcomes when patients are transitioned to oral antimicrobial therapy following an initial period of parenteral treatment $[22,23]$. Our study was conducted prior to recent publications and retrospective review precludes optimal assessment of treatment options as the decision to use oritavancin was based solely on ID physician progress notes. Additionally, we used oritavancin in socio-economic situations that may preclude use of oral antibiotics due to compliance, and the types of patients included in our study were not amenable to oral therapy based on the inclusion criteria. These limitations may also impact the ROI calculation; we were unable to predict the impact of oral therapy switch in the ROI calculation.

Multiple studies show a positive financial impact associated with oritavancin use. Lodise et al. reported the economic impact of using oritavancin in the emergency department compared to inpatient vancomycin administration using a cost-minimization model [18]. Jensen et al. used a decision analytic model and demonstrated a significant cost reduction to US payers and hospitals with single-dose oritavancin for ABSSSI [19, 20]. Cost reductions were attributed to saved hospital days and reduced outpatient drug administration costs. We present a simplified cost avoidance analysis for a small subgroup of patients where inpatient oritavancin administration facilitated early discharge and hospital days avoided. This analysis presumes all patients complete outpatient therapy without complications and could not complete treatment with an enteral antibiotic. Oritavancin was added to the formulary in our hospital system and restricted to use by infectious diseases consultation or to facilitate discharge due to socio-economic issues. Patients receiving oritavancin at discharge were medically ready for discharge and would have required inpatient treatment to complete therapy. Inpatient oritavancin administration was most cost effective for high reimbursement indications, such as surgical wound infections and endocarditis; oritavancin use typically did not result in a positive ROI when it was administered for ABSSSIs, regardless of when it was administered within the hospital course. While a more complex cost analysis is needed to further understand the implications of private insurance and off-label oritavancin utilization for complex Gram-positive infections specifically, we believe that early pharmacist involvement, in coordination with casemanagement colleagues and infectious diseases consulting teams, can identify patients poised to receive maximal cost benefit from inpatient oritavancin administration [21].

Oritavancin use at our institution required extensive, interdisciplinary coordination between the ID consult service, the patient's primary service, and antimicrobial stewardship pharmacists. We strive to administer the medication in an outpatient infusion center whenever possible. Insurance coverage and outpatient feasibility was investigated with the help of case management and social workers as soon as oritavancin was identified as a treatment option. The use of oritavancin in both the inpatient and the outpatient setting should include oversight from antimicrobial stewardship practitioners and or ID-trained clinicians due to the importance of balancing antimicrobial stewardship efforts and socioeconomic considerations. Care coordination resources may not be feasible for other hospitals; however, the significant cost savings realized by administering oritavancin to facilitate discharge and outpatient therapy could be resourced to support such a program.

\section{Conclusion}

In our case series of 75 patients, oritavancin treatment was found to be safe and effective for the treatment of complex Gram-positive infections. Inpatient administration may be 
considered in select patients to facilitate discharge and may be associated with cost avoidance. Effective, appropriate oritavancin use requires a multidisciplinary team in both inpatient and outpatient settings.

Author contributions All authors had a role in the study design and in conceiving and writing the manuscript. According to the guidelines of the International Committee of Medical Journal Editors (ICMJE, https ://www.icmje.org), all authors met the criteria for authorship and no deserving authors have been omitted.

\section{Compliance with Ethical Standards}

Funding No funding was received for the conduct of this study or the preparation of this manuscript. This manuscript is part of a supplement wholly funded by Melinta Therapeutics.

Conflict of interest LTS has received compensation for Speaker's Bureau activities from Melinta Therapeutics. LB, MA, EKM, JPV, EJE, and ERJ have no conflicts of interest to disclose.

Ethical statement This study was deemed quality assurance/improvement by the local institutional review board as it posed minimal risk to patients in the study. The study was performed in accordance with the ethical standards of the Declaration of Helsinki.

Data sharing The datasets generated and/or analyzed during the current study are available from the corresponding author on reasonable request.

Open Access This article is licensed under a Creative Commons Attribution-NonCommercial 4.0 International License, which permits any non-commercial use, sharing, adaptation, distribution and reproduction in any medium or format, as long as you give appropriate credit to the original author(s) and the source, provide a link to the Creative Commons licence, and indicate if changes were made. The images or other third party material in this article are included in the article's Creative Commons licence, unless indicated otherwise in a credit line to the material. If material is not included in the article's Creative Commons licence and your intended use is not permitted by statutory regulation or exceeds the permitted use, you will need to obtain permission directly from the copyright holder.To view a copy of this licence, visit http://creativecommons.org/licenses/by-nc/4.0/.

\section{References}

1. Liu C, Bayer A, Cosgrove SE, et al. Clinical practice guidelines by the Infectious Diseases Society of America for the treatment of methicillin-resistant Staphylococcus aureus infections in adults and children: executive summary. Clin Infect Dis. 2011;52:285-92.

2. Rybak MJ, Lomaestro BM, Rotschafer JC, et al. Therapeutic monitoring of vancomycin in adults: summary of consensus recommendations from the American Society of Health-System Pharmacists, the Infectious Diseases Society of America, and the Society of Infectious Diseases Pharmacists. Pharmacotherapy. 2009;29:1275-9.

3. Jeffres MN. The whole price of vancomycin: toxicities, troughs, and time. Drugs. 2017;77:1143-54.

4. Corey GR, Kabler H, Mehra P, et al. Single-dose oritavancin in the treatment of acute bacterial skin infections. N Engl J Med. 2014;370:2180-90.
5. Ralph Corey G, Good S, Jiang H, et al. Single-dose oritavancin versus 7-10 days of vancomycin in the treatment of gram-positive acute bacterial skin and skin structure infections: the SOLO II noninferiority study. Clin Infect Dis. 2015;60:254-62.

6. Syed YY, Scott LJ. Oritavancin: a review in acute bacterial skin and skin structure infections. Drugs. 2015;75:1891-902.

7. Datta R, McManus D, Topal J, Juthani-Mehta M. Long-acting lipoglycopeptides for gram-positive bacteremia at the end of life to facilitate hospice care: a report of 3 cases. Open Forum Infect Dis. 2018;5:15-7.

8. Johnson JA, Feeney ER, Kubiak DW, Corey GR. Prolonged use of oritavancin for vancomycin-resistant Enterococcus faecium prosthetic valve endocarditis. Open Forum Infect Dis 2015;2:ofv156.

9. Schulz LT, Dworkin E, Dela-Pena J, Rose WE. Multiple-dose oritavancin evaluation in a retrospective cohort of patients with complicated infections. Pharmacotherapy. 2018;38:152-9.

10. Werth BJ, Jain R, Hahn A, et al. Emergence of dalbavancin nonsusceptible, vancomycin-intermediate Staphylococcus aureus (VISA) after treatment of MRSA central line-associated bloodstream infection with a dalbavancin- and vancomycin-containing regimen. Clin Microbiol Infect. 2018;24:429.e1-.e5.

11. Naranjo CA, et al. A method of estimating the probability of adverse drug reactions. Clin Pharmacol Ther. 1981;30:239-45.

12. Kaiser Family Foundation Hospital Adjusted Expenses per Inpatient Day. https://www.kff.org/health-costs/state-indic ator/expenses-per-inpatient-day/?currentTimeframe $=0 \&$ sortM odel=\%7B \%22colId $\% 22: \% 22$ Location $\% 22, \% 22$ sort $\% 22: \% 22$ asc $\% 22 \% 7 \mathrm{D}$. Accessed 1 Oct 2018

13. Centers for Medicare and Medicaid Services. Inpatient charge data FY 2015. https://www.cms.gov/Research-Statistics-Dataand-Systems/Statistics-Trends-and-Reports/Medicare-ProviderCharge-Data/Inpatient2015.html. Accessed 11 May 2018.

14. United States Bureau of Labor. Inflation calculator. https://www. bls.gov/data/inflation_calculator.htm. Accessed 1 Oct 2018.

15. Stewart CL, Turner MS, Frens JJ, Snider CB, Smith JR. Realworld experience with oritavancin therapy in invasive gram-positive infections. Infect Dis Ther. 2017;6:277-89.

16. Delaportas DJ, Estrada SJ, Darmelio M. Successful treatment of methicillin susceptible Staphylococcus aureus osteomyelitis with oritavancin. Pharmacotherapy. 2017;37:e90-e9292.

17. Ho J, Archuleta S, Sulaiman Z, Fisher D. Safe and successful treatment of intravenous drug users with a peripherally inserted central catheter in an outpatient parenteral antibiotic treatment service. J Antimicrob Chemother. 2010;65:2641-4.

18. Lodise TP, Fan W, Sulham KA. Economic impact of oritavancin for the treatment of acute bacterial skin and skin structure infections in the emergency department or observation setting: cost savings associated with avoidable hospitalizations. Clin Ther. 2016;38:136-47.

19. Jensen IS, Wu E, Fan W, et al. Use of oritavancin in moderate-to-severe ABSSSI patients requiring IV antibiotics: a U.S. payer budget impact analysis. J Manag Care Spec Pharm 2016;22:752-64.

20. Jensen IS, Lodise TP, Fan W, et al. Use of oritavancin in acute bacterial skin and skin structure infections patients receiving intravenous antibiotics: a US hospital budget impact analysis. Clin Drug Invest. 2016;36:157-68.

21. Bounthavong M, Hsu DI, Okamoto MP. Cost-effectiveness analysis of linezolid vs. vancomycin in treating methicillin-resistant Staphylococcus aureus complicated skin and soft tissue infections using a decision analytic model. Int J Clin Pract 2009;63:376-86.

22. $\mathrm{Li} \mathrm{H}-\mathrm{K}$ et al. Oral versus intravenous antibiotics for bone and joint infection. N Engl J Med. 380.5 (2019):425-36.

23. Iversen $\mathrm{K}$ et al. Partial oral versus intravenous antibiotic treatment of endocarditis. N Engl J Med 380.5 (2019):415-24. 